\title{
Acute placental infection due to Klebsiella pneumoniae: Report of a unique case
}

\author{
SALWA S. SHEIKH ${ }^{1}$, SAMIR S. AMR ${ }^{1}$, \& JANICE M. LAGE ${ }^{2}$ \\ ${ }^{1}$ Dhahran Health Center, Pathology Services Division, Dhahran, Saudi Arabia, and ${ }^{2}$ Medical College of University of South \\ Carolina, Charleston, SC, USA
}

(Received 12 May 2004; revised 16 fuly 2004; accepted 9 November 2004)

\begin{abstract}
A 40-year-old woman, gravida 9, with seven healthy children and a history of one abortion (p $7+1)$, presented at 18 weeks of gestation with fever and malodorous vaginal discharge. Ultrasound revealed a macerated fetus. The placenta showed acute chorioamnionitis and acute villitis with microabscess formation. Blood and vaginal cultures both grew Klebsiella pneumoniae. This is the first reported case in English literature of Klebsiella pneumoniae causing suppurative placentitis leading to fetal demise.
\end{abstract}

Keywords: Placenta, chorioamniotic membranes, infection, Klebsiella

\section{Introduction}

Prenatal infection can induce a variety of pathologic changes within the placenta. The placenta can be affected by maternal infection either by a systemic route or via an ascending amniotic fluid infection. Various organisms, including bacteria, viruses, protozoa and fungi, can infect the placenta. Some infections tend to cause specific gross and microscopic changes of the placenta, whereas others show no characteristic features. Bacterial infections usually lead to an acute suppurative response, whereas viruses and fungi, in general, elicit a more chronic inflammatory response $[1,2]$. We report a unique case of acute placentitis caused by Klebsiella pneumoniae, leading to intrauterine fetal death.

\section{Case report}

A 40-year-old woman, gravida 9 , para $7+1$, presented to our hospital with a 2-day history of vaginal discharge which later turned green and was associated with vaginal bleeding of 1 day's duration. In addition, she had fever, dizziness, weakness, nausea and vomiting, but no abdominal pain. On clinical examination, she was febrile $\left(41^{\circ} \mathrm{C}\right)$ and looked ill. Her abdomen was not tender and the size of her uterus corresponded with 16 to 18 weeks of gestation. Vaginal examination revealed a malodorous blood-stained discharge. The cervix was dilated by $3 \mathrm{~cm}$ and the fetal head could be felt. Transabdominal US showed evidence of fetal death with a collapsed fetal skull.

The woman had marked leukocytosis (white cell count 30300 , neutrophils $70 \%$ ). Treatment with ampicillin, gentamycin, and oral flagyl was initiated. She also received oxytocin and responded rapidly with expulsion of a dead, macerated male fetus of 18 weeks of gestation by weight and size. Both blood and vaginal cultures grew Klebsiella pneumoniae which was resistant to ampicillin and was replaced by Cephaclor. The next day the woman's condition improved dramatically; she became afebrile and was feeling much better. Her white cell count came down to 7600 within 2 days. She was followed-up in the clinic for 24 months and continues to do well.

\section{Pathological findings}

The placenta weighed $113 \mathrm{~g}$ (normal placental weight for 16 to 18 weeks of gestation is 80 to $112 \mathrm{~g}$ ) [1], and measured $10 \times 7 \times 2 \mathrm{~cm}$. The fetal

Correspondence: Salwa S. Sheikh, Saudi Aramco, P.O. Box 12113, Dhahran 31311, Saudi Arabia. Tel: 877-8109. Fax: 877-3791.

E-mail: salwa.sheikh@aramco.com

ISSN 1064-7449 print/ISSN 1098-0997 online (C) 2005 Taylor \& Francis Group Ltd DOI: $10.1080 / 10647440400028177$ 
membranes were dull and the fetal surface showed blue-gray discoloration. The maternal surface showed intact cotyledons. The fetus weighed $138 \mathrm{~g}$ and measured $13.5 \mathrm{~cm}$ crown to rump and $21 \mathrm{~cm}$ crown to heel (normal fetal weight for 16 to 18 weeks of gestation is 130 to $250 \mathrm{~g}$ ) [1]. No gross congenital malformations were noted. No autopsy was performed.

On histologic examination, the umbilical cord was trivascular. There was acute funisitis with numerous bacilli permeating the Wharton's jelly. Bacilli were also present within the lumina of the umbilical cord blood vessels, admixed with fibrin and blood. The fetal membranes showed acute chorioamnionitis and were heavily permeated by bacilli. Sections of the placenta showed extensive acute, suppurative villitis with micro-abcess formation (Figure 1). In addition to the presence of numerous neutrophils, a minor component of the inflammatory infiltrate was mononuclear. The fetal capillaries within the villi were loaded with bacilli that streamed into the connective tissue cores of the villi and eventually into the intervillous space (Figure 2). There was marked,

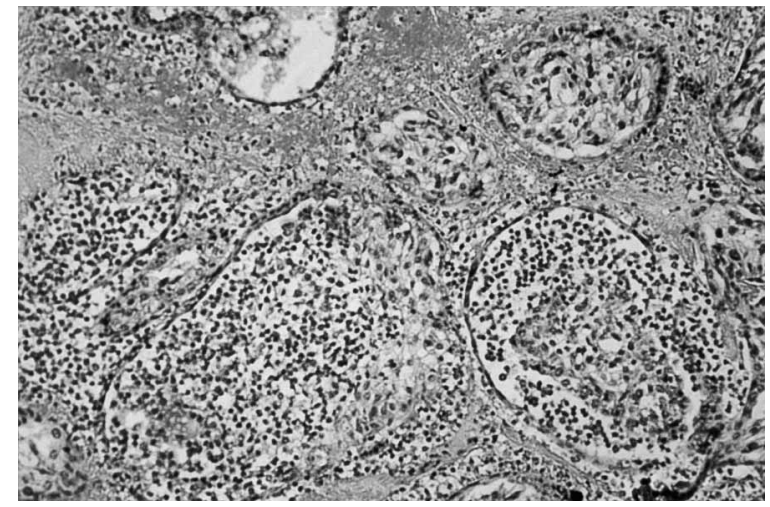

Figure 1. Low-power view of placenta exhibiting chorionic villi heavily infiltrated by numerous neutrophils.

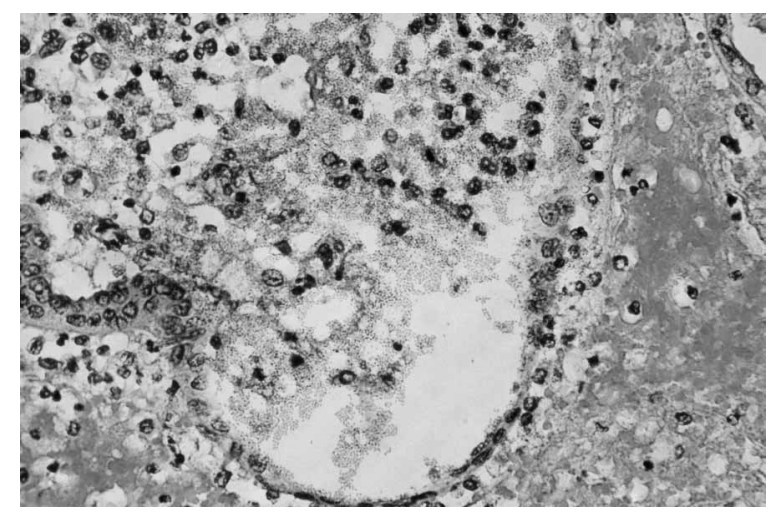

Figure 2. High-power view of a chorionic villus showing numerous bacilli and neutrophils. acute intervillositis with maternal neutrophils clearly invading through the trophoblastic covering of the villi and into the villous stroma. Tissue Gram stain showed the bacilli to be Gram-negative rods.

\section{Discussion}

Acute chorioamnionitis is usually caused by ascending infection that can precipitate premature rupture of membranes and delivery. In many cases of placental infection, the organisms reach the placenta from the maternal circulatory system; this hematogenous infection involved the placental parenchyma itself rather than the membranes. As infection progresses, it may extend to the membranes. The hallmark of hematogenous infection is villitis, an inflammatory lesion within the villous substance. However, this is a rather arbitrary classification that simply demarcates the progress of an inflammatory process, and the findings may vary depending upon the stage or severity of inflammation and the causative agent. One important factor to consider is that the vaginally delivered placenta has just passed through a heavily colonized birth canal, making it extremely difficult to decide whether the organisms cultured from the placenta have been acquired while in the uterus or were contaminants from the vagina $[1,2]$.

Villitis may be acute or chronic and may be focal, diffuse, or necrotizing. The acute form tends to be associated with infection that is usually bacterial in origin. Although chronic villitis can occur secondary to a viral or parasitic infection, it often presents as villitis of unknown etiology (VUE). This entity remains a significant challenge to the pathologist, but a number of possibilities are entertained, including viral disease by an unrecognized agent that reflects some sort of immune reaction, or preeclampsia. At the present time, there is no conclusive evidence of an etiologic agent causing VUE $[1,2]$.

The bacteria most commonly isolated from cases of chorioamnionitis are Escherichia coli, coagulase positive Staphylococcus, Hemophilus vaginalis, Streptococci, Listeria monocytogenes and other Gram-negative bacilli. The frequent presence of Mycoplasma in the birth canal and the ability of the organism to cause chorioamnionitis are well documented $[1,2,3]$.

Certain histologic features are commonly seen with specific organisms $[1,4]$. Bacterial infection, as expected, leads to a neutrophilic infiltrate. Gramnegative bacilli, especially Eschericia coli, may cause chorioamnionitis due to ascending infection, which is associated with neonatal meningitis. Placental infection by pyogenic organisms, such as coagulasepositive Staphylococcus and Streptococcus, is usually by an ascending route of infection rather than by hematogenous spread. Infection with group B 
Streptococci may lead to frequent perinatal complications, and it is important to recognize such infection early on, since the effects of this infection can be devastating to the fetus and the newborn [5]. Streptococcus pneumoniae and Hemophilus influenzae can cause fatal maternal and serious fetal infections. Other bacterial organisms reported, which are etiologically related to placental infection, are Salmonella, Shigella, Clostridium perfringes, Diphtheroids, Fusobacterium, Bacteroides fragilis, Streptobacillus moniliformis, Gardnerella vaginalis, Francisella tularensis and Brucella species. Listeria monocytogenes leads to a heavy placenta with septic intervillositis with microabscess and, rarely, macro-abscess formation. Treponema pallidum also results in a heavy placenta with a relative villous immaturity for age, acute or chronic lymphoplasmacytic villitis, obliterative changes of the villous vessels, avascular villi, necrotizing funisitis, and occasionally granulomatous villitis [6]. Campylobacter infection leads to macro-abcesses, perivillous fibrin masses, and widespread acute villitis. Placentas with Mycobacterium tuberculosis exhibit an abnormal decidual surface with decidual necrosis and granuloma formation. Neisseria gonorrheae may also occasionally lead to acute chorioamnionitis $[1,2]$.

Although many bacteria have been reported as the culprit organisms in the etiology of placentitis, to our knowledge, there is no single case in the literature of acute placental infection caused by Klebsiella pneumoniae. The Klebsiella genus belongs to the family Enterobacteriaceae, a large heterogenous group of Gram-negative bacilli. Four species belong to the Klebsiella genus, the most significant being $K$. pneumoniae, which behaves as an opportunistic or nosocomial pathogen. Klebsiella species are an important component of the normal flora of gastrointestinal tract. They cause a wide variety of clinical illnesses including pneumonia, urinary tract infections, bacteremia and a chronic granulomatous disease of the upper airways [7]. It is important to recognize this organism as a possible etiologic agent of acute placentitis, in order to provide early and prompt treatment to the infected mother and to avoid complications.

Various viruses can also lead to placental infection. Cytomegalovirus is one of the most frequent causes of intrauterine infection in humans [8]. Histologically there is lymphoplasmacytic chronic villitis, focal villous necrosis with neutrophilic infiltration, atrophy and fibrosis. These represent 1 to $2 \%$ of cases of chronic villitis. Characteristic viral inclusions may be difficult to demonstrate in many cases, and thus a proportion of these cases are underdiagnosed. Polymerase chain reaction, in-situ hybridization, and immunohistochemistry techniques are helpful tools in confirming the diagnosis. Herpes simplex virus, when infecting by the hematogenous route, can lead to necrosis of trophoblasts of scattered villi without any significant inflammatory response or can cause necrotic abscesses in the intervillous spaces with marked karyorrhexis, degeneration and neutrophilic inflammation [2, 9]. Varicella herpes zoster infection can cause widespread necrotizing villitis, chronic villitis that may contain obliteration of fetal vessels, and necrotizing granulomata in some villi with multinucleated giant cells [10]. The effect of HIV type 1 on the placenta and the role of the placenta in mother-to-child HIV-1 transmission are not well understood. A recent study showed that HIV-infected women are more likely than HIV-uninfected women to develop chorioamnionitis and deciduitis, and are less likely to have villitis, but placental inflammatory lesions are not associated with increased perinatal HIV transmission [11].

Mycotic infections of placenta are rare and are nearly always caused by Candida, most often by ascending infection. The placenta can be infected by parasites and protozoa. Toxoplasma gondii often produces chronic villitis, increased numbers of Hofbauer cells, fibrosis and sometimes necrosis and granulomatous inflammation. The fetal vessels often show endarteritis. Encysted and free forms of Toxoplasma can be seen, in the chorionic plate rather than in the villi $[1,2]$. Trypanosoma cruzi characteristically shows chronic villitis consisting mostly of CD8-positive $\mathrm{T}$ cells and macrophages. The organisms are usually seen in the villous tissue [12].

Mycoplasmas are a well recognized group of pathogens of many animals, and are known urogenital pathogens for humans. The relationship of Mycoplasma to premature rupture of membranes and chorioamnionitis is still debated, as different studies show conflicting results. In general, the impression is that the genital mycoplasmas are a frequent cause of chorioamnionitis and premature birth. Interestingly it is the commonest organism to be cultured from placentas at Cesarian section in cases with ruptured membranes $[1,3]$.

Our patient presented with no history of repiratory illness, urinary tract infection, instrumentation or hospitalization. She initially had vaginal symptoms that led to a more systemic infection. The vaginal as well as blood cultures both grew Klebsiella pneumoniae. In all probability this represented an ascending infection that was severe enough to cause extensive villitis in addition to chorioamnionitis.

Recently, a case of ascending infection of uterine leiomyoma during pregnancy was reported in a 44year-old woman who presented in her 26th week of gestation. Culture showed the culprit organism to be Klebsiella pneumoniae that led to pyomyoma. A 
Cesarian section with myomectomy confirmed the diagnosis. There were no signs of infection or other pathological findings in the evaluation of placenta and membranes [13].

In conclusion, numerous organisms that may lead to placental infection, whether bacterial, viral or parasitic, have been reported and studied. To the best of our knowledge, this is the first reported case of acute placental infection secondary to Klebsiella pneumoniae causing suppurative villitis and resulting in intrauterine death of an 18-week-old male fetus.

\section{Acknowledgment}

The authors wish to acknowledge the use of Aramco Medical Services Organization (SAMSO) facilities for the research data used in this study. Opinions expressed in this article are those of the authors and not necessarily of the SAMSO.

\section{References}

1. Benirschke K, Kaufmann P. Infectious diseases. In: Pathology of the human placenta. 4th ed. New York: Springer-Verlag; 2000. pp 591-659.

2. Fox H. Infection of the placenta. In: Major problems in pathology. Vol 7: Pathology of the placenta. W. B. Saunders, Philadelphia; 1978. pp 286-325.

3. Shurin P, Alpert S, Rosner B, Driscoll S, Lee Y. Chorioamnionitis and colonization of the newborn infant with genital mycoplasmas. N Engl J Med 1975;293(1):5-8.
4. Baldwin VJ. Placenta. In: Dimmick JE, Kalousek DK, editors. Developmental pathology of the embryo and fetus. Philadelphia: Lippincott; 1992. pp 271-319.

5. Larsen JR, London WT, Baker CJ, Curfman BL, Sever JL. Intraamniotic infection due to group G Streptococcus: treatment and antibody response. Obstet Gynecol 1981;58(2): 222-226.

6. Sheffield J, Sanchez P, Wendel G, et al. Placental histopathology of congenital syphilis. Obstet Gynecol 2002;100:126-133.

7. Schwartz DA, Geyer SJ. Klebsiella and rhinoscleroma. In: Conner DH, Chandler F, Schwartz D, Manz H, Lack E, editors Pathology of infectious diseases. Stanford, Connecticut: Appleton and Lange; 1997. pp 589-595.

8. Saetta A, Agapitos E, Davaris P. Determination of CMV placentitis. Diagnostic application of the polymerase chain reaction. Virchows Arch 1998;432:159-162.

9. Chatterjee A, Chartrand S, Harrison C, Felty-Duckworth A, Bewtra C. Perinatal/neonatal case presentation. Severe intrauterine Herpes simplex disease with placentitis in a newborn of a mother with recurrent genital infection at delivery. J Perinatol 2001;21:559-564.

10. Petignat P, Vial Y, Laurini R, Hohlfeld P. Fetal Varicellaherpes zoster syndrome in early pregnancy: ultrasonographic and morphological correlation. Prenat Diagn 2001;21:121124.

11. Schwartz D, Sungkarat S, Shaffer N, et al. Placental abnormalities associated with human immunodeficiency virus type 1 infection and perinatal transmission in Bangkok, Thailand. J Infect Dis 2000;182:1652-1657.

12. Altemani A, Bittencourt A, Lana A. Immunohistochemical characterization of the inflammatory infiltrate in placental Chaga's disease: a qualitative and quantitative analysis. Ann Trop Med Hyg 2000;62:319-324.

13. Grune B, Zikulnig E, Gembruch U. Sepsis in second trimester of pregnancy due to an infected myoma. a case report and review of the literature. Fetal Diagn Ther 2001;16:245-247. 


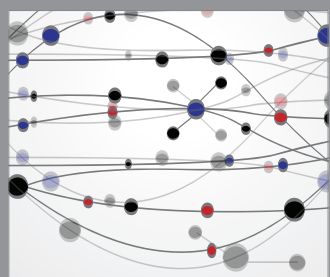

The Scientific World Journal
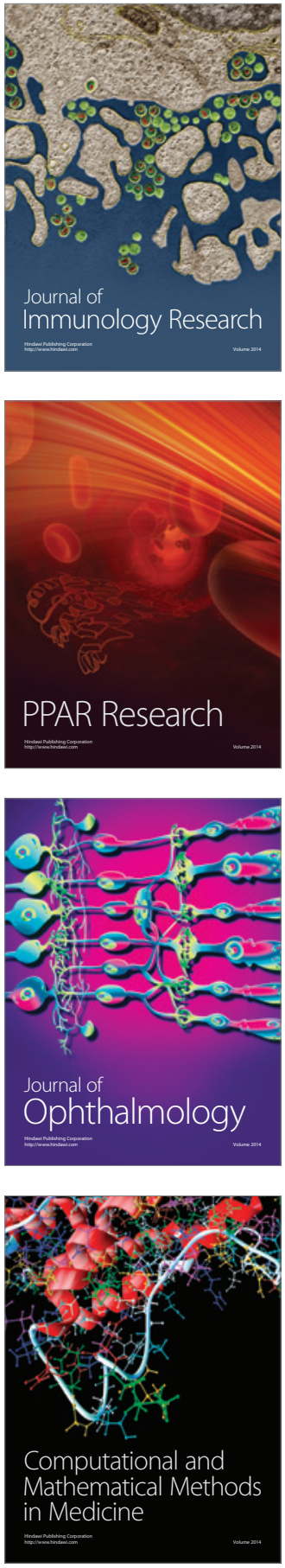

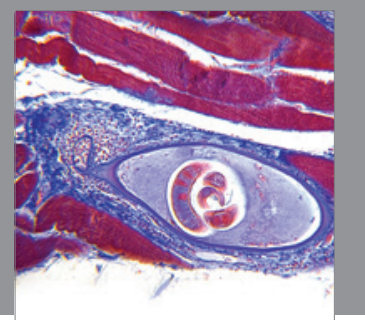

Gastroenterology

Research and Practice
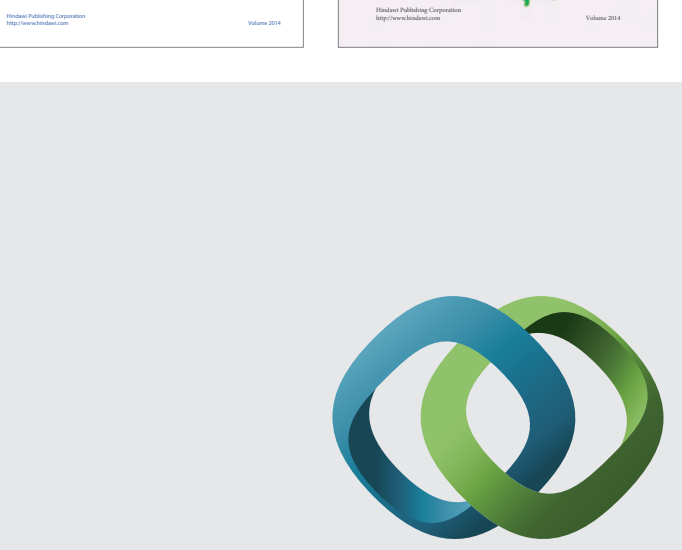

\section{Hindawi}

Submit your manuscripts at

http://www.hindawi.com
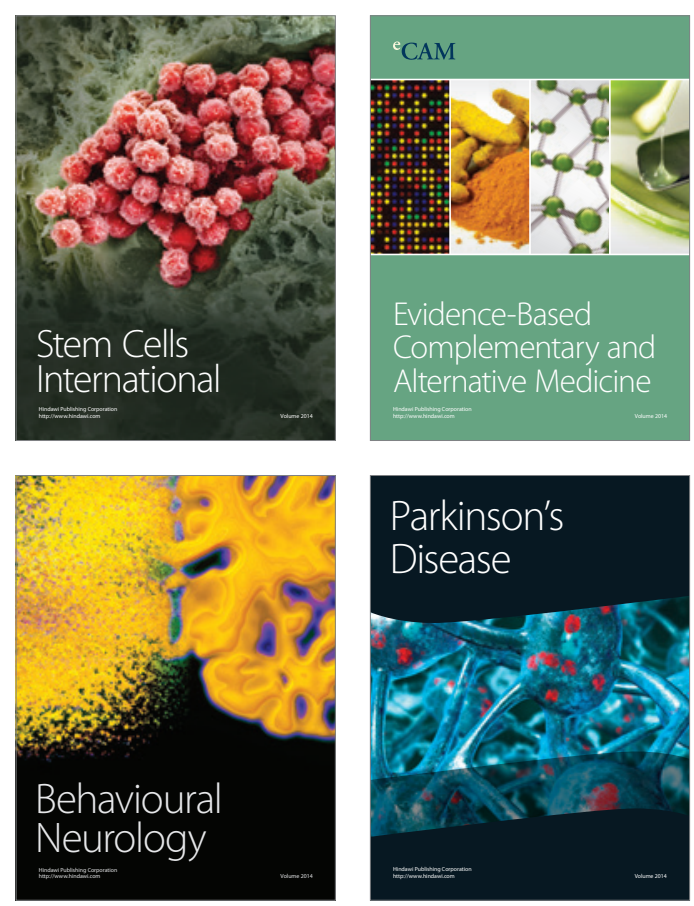

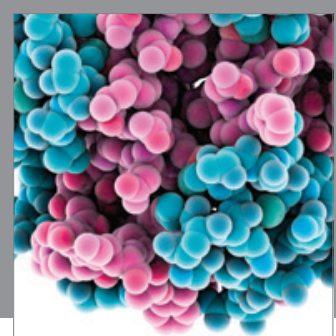

Journal of
Diabetes Research

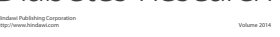

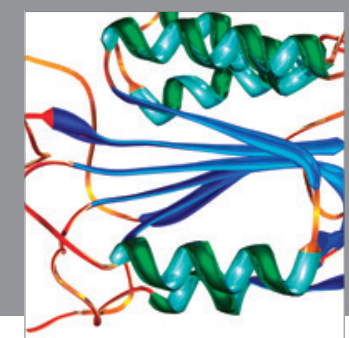

Disease Markers
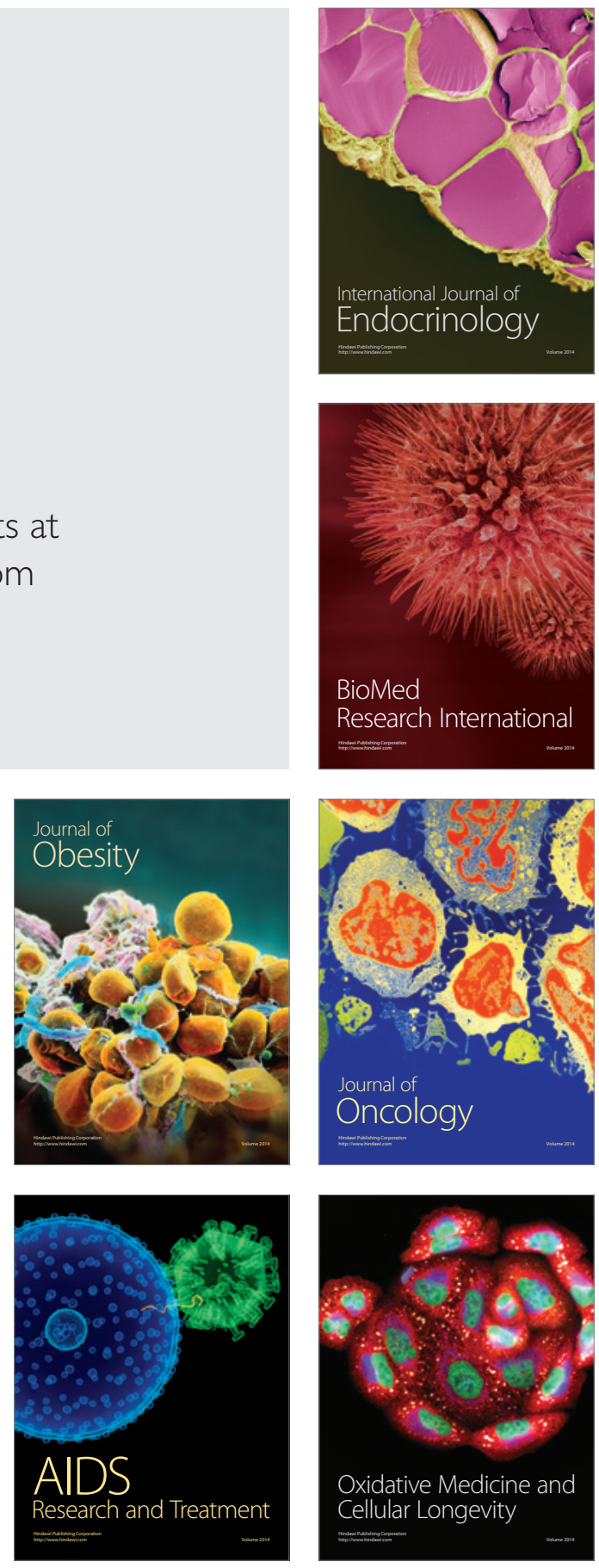